\title{
Calidad de las clases de pediatría comunitaria desde la percepción del estudiante, luego de la aplicación de conocimientos neurocientíficos
}

\author{
Medical students' perception of community pediatrics lessons quality following application
}

of neuroscientific knowledge

\author{
Manuel Hernán Izaguirre Sotomayor ${ }^{1, a}$, Luis Américo Reátegui Guzmán ${ }^{1, b}$ \\ Departamento de Pediatría, Facultad de Medicina San Fernando, Universidad Nacional Mayor de San Marcos, Lima, Perú. \\ ${ }^{a}$ Profesor Principal; ${ }^{b}$ Profesor Asociado.
}

\begin{abstract}
Resumen
Introducción: Las investigaciones realizadas en las últimas décadas sobre el rol del cerebro en el proceso educativo han aportado las bases neuronales del aprendizaje y otras funciones cerebrales que son estimuladas en el aula. Objetivos: Evaluar la calidad del servicio educativo en la rotación de Pediatria Comunitaria desde la perspectiva del estudiante luego de la aplicación de conocimientos neurocientíficos. Diseño: Estudio transversal, descriptivo de diseño cuantitativo. Lugar: Departamento de Pediatría, Facultad de Medicina San Fernando, Universidad Nacional Mayor de San Marcos, Lima, Perú. Participantes: Estudiantes de Pediatría Comunitaria. Intervenciones: Aplicación de la encuesta ServPerf. Resultados: La satisfacción global para todas las dimensiones fue de satisfacción, siendo el nivel de insatisfacción mínima. La satisfacción media para todas las dimensiones fue de satisfacción, a excepción de la dimensión entorno del aprendizaje, que fue de insatisfacción. El atributo el conocimiento brindado en clase, tuvo conexión evidente con la realidad; fue el de mayor prioridad. Conclusiones: La utilización de los conocimientos neurocientíficos mejora la percepción de la calidad del servicio educativo. La dimensión que alcanzó más alta satisfacción media fue materiales didácticos seguidos por información recibida e integrada. El conocimiento brindado en clase tiene conexión evidente con la realidad, tuvo la más alta prioridad.
\end{abstract}

Palabras clave: Calidad percibida; dimensiones de la calidad, conocimientos neurocientíficos.

Abstract

Introduction: In the last few decades research on the role of the brain in the educational process has provided the neuronal basis of learning and other brain functions that are stimulated in the classroom. Objectives: To determine the quality of education service from the perspective of the student on the application of neuroscientific knowledge during Community Pediatrics rotation. Design: Cross sectional, descriptive quantitative study. Setting: Pediatrics Department, San Fernando Medical School, Universidad Nacional Mayor de San Marcos, Lima, Peru. Participants: Students of Community Pediatrics. Interventions: Application of Servperf survey. Results: Overall satisfaction and minimum level of dissatisfaction for all dimensions of satisfaction was found, with the exception of dissatisfaction in the learning environment dimension. The attribute knowledge provided in class had clear connection with reality; it showed the highest priority. Conclusions: The use of neuroscience enhances the perception of educational service quality. The dimension obtaining highest average satisfaction was teaching materials, followed by received and integrated information. The highest priority was obtained by knowledge provided in class has clear connection with the reality.

Keywords: Perceived quality, dimensions of quality, neuroscience knowledge.

An Fac med. 2014;75(2):145-9 / doi: http://dx.doi.org/10.15381/anales.v75i2.8378

\section{INTRODUCCIÓN}

El papel que el cerebro desempeña en el proceso educativo ha sido un área de énfasis de las investigaciones, principalmente en la última década. Benarós y $\mathrm{col}^{(1)}$ examinaron las implicancias de los vínculos entre neurociencia, psicología cognitiva y educación. Así mismo, Campos ${ }^{(2)}$ precisó que la neurociencia ha aportado las bases neuronales del aprendizaje, de la memoria, de las emociones y otras funciones cerebrales que son estimuladas y reforzadas a diario en el aula. Serra ${ }^{(3)}$ revisó el sustrato neural del procesamiento numérico y del cálculo y Gudiño ${ }^{(4)}$, así como Aparicio ${ }^{(5)}$, enfatizaron la casi nula participación de la neurociencia en la formación del docente, a pesar de la transdisciplinariedad de la educación.

Es conocido que la neurociencia ha evidenciado las diferencias en el aprendizaje según el género ${ }^{(6-9)}$, que el estrés agudiza la memoria en el corto plazo pero la altera en el largo plazo ${ }^{(10)}$, además de afectar severamente al hipocampo ${ }^{(11)}$. También, la capacidad de aprendizaje es afectada por la hipoglucemia y la deshidratación ${ }^{(12)}$. En los nativos informáticos, los estímulos visuales y auditivos activan al cerebro límbico ${ }^{(10)}$ y el cambio del entorno del aprendizaje despierta el interés y la emoción en el tema de la clase ${ }^{(11,12)}$.

Los estímulos musicales de baja frecuencia facilitan el desarrollo de procesos de atención, memoria y aprendizaje, así como la actividad física, el estado nutricional, los ambientes enriquecidos y el sueño favorecen la comprensión y el crecimiento de nuevas neuronas en el hipocampo ${ }^{(11-13)}$. Así mismo, la comunicación no verbal, las situaciones divertidas y emocionalmente positivas 
activan a las neuronas espejo ${ }^{(12-14)}$, así como la estimulación táctil estimula la neuroplasticidad cerebral ${ }^{(2,12,15,16)}$ que es modulada por la neurotrofina ${ }^{(17)}$. La ventilación, la iluminación y la temperatura adecuada mejoran el aprendizaje, así como el silencio mantiene la atención fija por más tiempo ${ }^{(12,17)}$.

Existen estudios donde la actividad psicomotriz favorece el desarrollo cognitivo y socio afectivo al optimizar su disposición psíquica al esfuerzo cognitivo ${ }^{(12)}$, que demanda la apropiación por parte del educando del concepto principal de la clase, explorarlo y comprenderlo ${ }^{(14)}$. La imitación y lo novedoso capturan la atención del hipocampo que, al valorarlo como interesante, lo almacena creando una representación neuronal de ella ${ }^{(11)}$, así como la emoción positiva facilita lo aprendido al incrementar la liberación de dopamina ${ }^{(4)}$.

Existe estrecha relación entre emoción y cognición ${ }^{(11,12)}$, dado que se recuerda lo memorizado en un contexto emocional positivo al activarse el hipocampo. La relación del contexto y el aprendizaje deviene del hipocampo y la amígdala ${ }^{(11)}$, así como el aprendizaje contextual depende del núcleo accumbens, del estriado y otras áreas corticales ${ }^{(18)}$. Por otro lado, el sistema atencional supervisor actúa frente a tareas novedosas activando los procesos de anticipación, selección de objetivos, planificación y control, actuando la memoria de trabajo y el sistema atencional supervisor como el sistema ejecutivo central ${ }^{(19,20)}$.

La memorización con imágenes visuales estimula el hipocampo y la corteza parieto-témporo-occipital, siendo la capacidad de imitación importante para la enseñanza ${ }^{(13)}$. El sistema atencional operativo que trabaja con contenidos de la memoria de trabajo ${ }^{(21)}$ es necesario para el accionar del ejecutivo central, que realiza tareas cognitivas para ejecutar operaciones de selección de estrategias y medidas de control, así como de los objetivos y medios para alcanzarlos ${ }^{(2,22)}$. Finalmente, el marcador somático es necesario para el proceso de razonamiento al comparar posibles resultados y establecer ordenaciones para elaborar luego inferencias, expandiendo la atención por todo el sistema cognitivo ${ }^{(19-21)}$. Por lo tanto, la presente investigación intenta contribuir a la mejora de la calidad de la educación universitaria en el país.

\section{MÉTODOS}

El estudio fue transversal, descriptivo, correlacional de diseño cuantitativo. La muestra estuvo conformada por 113 estudiantes de la rotación de Pediatría Comunitaria, entre los meses de enero a diciembre de 2012. Las unidades de análisis estuvieron conformadas por los estudiantes de la rotación de Pediatría Comunitaria.

Para aplicar algunos de los conocimientos neurocientíficos que favorecen el aprendizaje, como evitar la hipoglucemia y la deshidratación, se permitió que los estudiantes ingirieran alimentos y líquidos en el aula. Además, se adecuó las estrategias didácticas al entorno habitual de aprendizaje, buscando que las actividades docentes superaran lo esperado por los estudiantes. Así mismo, se reforzó el aprendizaje a través de objetos, emociones y ejemplos de la vida real, para que los estudiantes corroboraran la utilidad de lo aprendido. También, se estimuló la percepción visual a través de colores y movimientos para facilitar el desarrollo de procesos de atención y memoria. Se insistió que el docente pusiera especial interés en la comunicación no verbal y que sonriera como estrategia para mantener la motivación, toda vez que activan las neuronas espejo; se utilizó los materiales didácticos adecuados.

Así mismo, se mejoró la ventilación y la temperatura del salón de clase; se evitó los ruidos para mantener la atención y se pasó música de fondo seguida con intervalos de silencio, para favorecer el aprendizaje y la comprensión lectora. También, se practicó ejercicios con las manos y los pies, además de realizar pequeños saltos antes y en el intermedio de cada clase; se priorizó lo novedoso para el estudiante para poder estimular al hipocampo y lograr emocionarlo, incrementando la liberación de dopamina.

También se priorizó, después de haber percibido el objeto de estudio, la evocación en clase; se estimuló a los estudiantes con los sentimientos y las historias de vida; se buscó siempre que los educandos estudiaran en un estado de buen humor y con ánimo positivo. Durante todo el proceso educativo se conversó con los estudiantes; se incentivó el uso de la tormenta de ideas como estrategia para eliminar el miedo; se trató de mejorar la atención, con el movimiento del profesor y volumen cambiante de la voz del docente al explicar los contenidos académicos.

Al no haberse utilizado métodos invasivos que pusieran en peligro la salud física y mental de los estudiantes, no existieron problemas éticos en la presente investigación. Sin embargo, se respetó los principios éticos aceptados por la sociedad y por los Comités de Ética de la universidad, así como la propiedad intelectual. Al procesar y comunicar los resultados, la identidad de los estudiantes fue reemplazada por códigos. El instrumento que se utilizó fue el SERVPERF ${ }^{(23)}$, que mide la calidad percibida en base a las percepciones del estudiante después de haber recibido el servicio educativo. Instrumento que ha sido ampliamente empleado para la evaluación de la calidad de los servicios en el Perú y el mundo.

Las dimensiones de la calidad percibida para la presente investigación fueron el entorno del aprendizaje, la información recibida e integrada, los materiales didácticos y la percepción del docente, las mismas que estuvieron basadas en los establecidos por Cronin y Taylor ${ }^{(23)}$. La interpretación de los niveles de satisfacción se realizó según los siguientes parámetros: satisfacción amplia (5); satisfacción moderada (4); satisfacción (3); insatisfacción leve / moderada (2); insatisfacción severa (1). 
La técnica utilizada fue la encuesta directa cara a cara. El instrumento utilizado para el recojo de los datos fue el SERPERF modificado, que fue aplicado por un encuestador ajeno al servicio y capacitado en el manejo y procedimiento de su aplicación. Los datos obtenidos fueron ingresados en Microsoft Office Excel 2000 y tabulados mediante software SPSS versión 15. Finalmente, se realizó el análisis descriptivo de todas las variables, a través de la determinación de proporciones de las variables socio demográficas y medias de satisfacción. En la ejecución de la investigación no se presentaron limitaciones importantes, salvo las inasistencias de los estudiantes al momento de responder la prueba. Lo que determinó la exclusión del estudio de varios estudiantes.

\section{RESULTADOS}

Los datos socio demográficos revelaron que los alumnos mayoritariamente tuvieron una edad entre 21 a 30 años, fueron varones, estudiaron a tiempo completo, estuvieron por debajo del tercio superior y acudieron solo desayunando.

El nivel de satisfacción global de los estudiantes respecto a la dimensión entorno del aprendizaje fue de satisfacción amplia (53\%), contrariamente al 9\% que mostró niveles de insatisfacción leve a moderada. La mayoría (más de $90 \%$ ) de los estudiantes encuestados informaron estar moderada y ampliamente satisfechos con la información recibida e integrada y con los materiales didácticos utilizados en clase. En cuanto al nivel de la satisfacción global respecto a la dimensión percepción del docente, la mayoría (más de $80 \%$ ) se encontró satisfecho amplia y moderadamente con la labor docente. El nivel de satisfacción global del servicio educativo en general, para la mayoría (más de $80 \%$ ) también fue de satisfacción amplia y moderada.

Por otro lado, el nivel de satisfacción media en las dimensiones información recibida e integrada, materiales didác- ticos y percepción del docente revelaron satisfacción con el servicio. Solo en el caso de la dimensión entorno del aprendizaje evidenció insatisfacción leve. Por otra parte, se observó niveles de satisfacción con el servicio educativo en general.

Con respecto a los atributos a los cuales los estudiantes les asignaron mayor prioridad fueron el conocimiento brindado en clase tiene conexión evidente con la realidad; el profesor genera diálogos abiertos para la búsqueda de conocimiento a través de las intervenciones, dándole al aprendizaje el rol protagónico; y el profesor se esmera en que percibamos y representemos mentalmente el objeto o tema central de la clase. Mientras que los atributos el salón de clase está ventilado y con temperatura adecuada para una óptima educación y los materiales utilizados en clase son visualmente atractivos y comprensibles tendieron a tener menor relevancia para los estudiantes.

\section{DISCUSIÓN}

Los resultados referidos a la calidad percibida del servicio educativo, al ser la población estudiada mayoritariamen- te masculina ${ }^{(7-9)}$ y no haber alcanzado mayores niveles de satisfacción global, se explicaría por no haberse individualizado las estrategias didácticas en función de los 'perfiles pedagógicos' de los educandos ${ }^{(1,14)}$. Por otro lado, el estrés permanente que la gran mayoría presentó por utilizar el transporte público y tener tareas personales y académicas le habría generado dificultades para el aprendizaje ${ }^{(10)}$, al alterar la cognición y el cableado neuronal del hipotálamo ${ }^{(11)}$. Otra situación que explicaría los resultados obtenidos fue el hecho que la mayoría de los estudiantes solo había desayunado, y al ser la capacidad de aprendizaje dependiente de energía se habría afectado por la hipoglucemia producida ${ }^{(12)}$. Así mismo, los educandos exigen acceder a estímulos visuales y auditivos que le deparen recompensa inmediata en respuesta a su cerebro límbico ${ }^{(10)}$.

En el caso del nivel de satisfacción global más bajo para la dimensión entorno del aprendizaje se explicaría por el hecho que, por motivos económicos, no se adecuó el ambiente con novedades para lograr despertar el interés y la emoción en el tema de la clase ${ }^{(12)}$. Sin embargo, se buscó que las actividades docentes superaran a lo esperado por

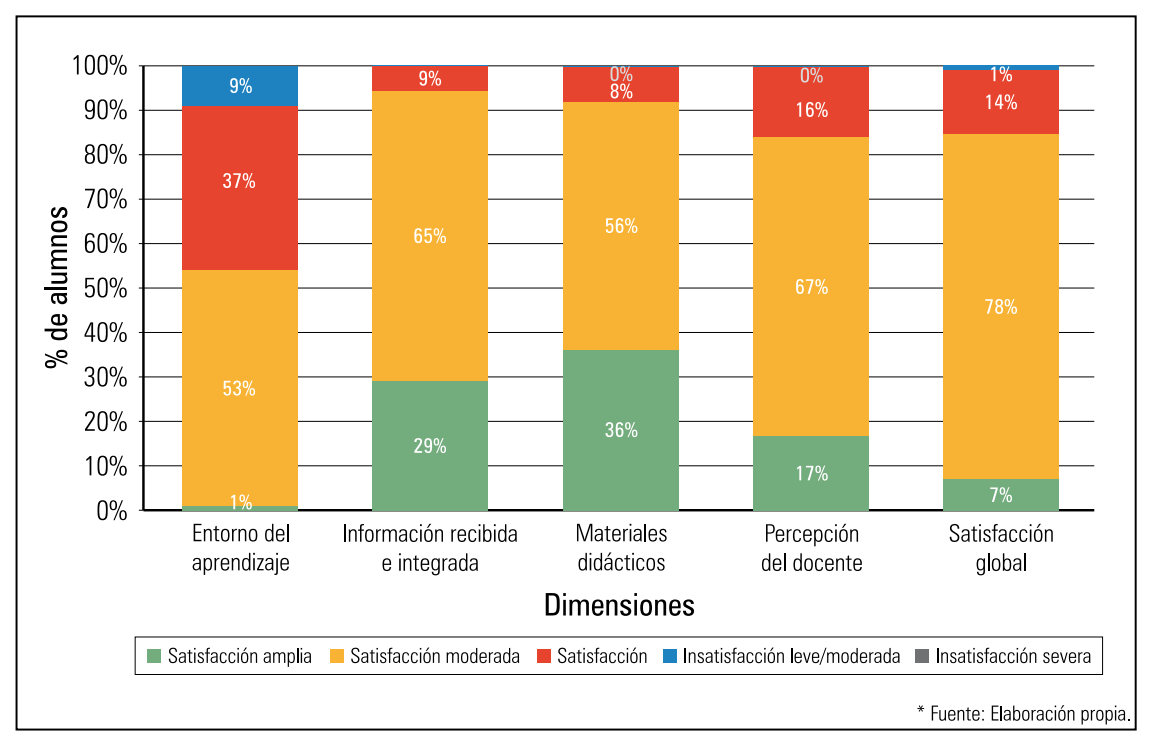

Figura 1. Satisfacción global y por dimensiones de la calidad del servicio educativo post aplicación de la Neurociencia. Rotación de Pediatría Comunitaria. UNMSM - 2012. 
los estudiantes, dado que se aprende más con la realización de experiencias positivas ${ }^{(11)}$. Se reforzó el aprendizaje a través de emociones y ejemplos de la vida real; se estimuló la percepción visual; se usó la actividad física, los ambientes enriquecidos, la intuición, la gimnasia cerebral y el sueño para facilitar la comprensión ${ }^{(13)}$ y para estimular el crecimiento de nuevas neuronas, especialmente en el hipocampo ${ }^{(12)}$, que se constituye en el profesor del córtex cerebral durante el sueño ${ }^{(11)}$.

En la dimensión información recibida e integrada se obtuvo un nivel de satisfacción global mayor que en la dimensión entorno del aprendizaje, gracias a que el docente puso especial interés en la comunicación no verbal que activa a las neuronas espejo. Se estimuló a los estudiantes con imágenes positivas y agradables sobre el tema de cada clase ${ }^{(12)}$. La dimensión materiales didácticos fue el de mayor nivel de satisfacción, debido probablemente al uso de materiales didácticos adecuados que estimulan la neuroplasticidad cerebral ${ }^{(2,12,15,16)}$. Precisamente la aplicación de los conocimientos neurocientíficos al proceso de aprendizaje lo hace más efectivo y significativo ${ }^{(22)}$, dado que la adquisición de conocimientos y de habilidades es influenciada por las habilidades intelectuales, la organización cualitativa del conocimiento y la motivación ${ }^{(24)}$. Así mismo, el mundo globalizado exige que los educandos desarrollen el pensamiento creativo y práctico ${ }^{(14)}$, dado que la neurotrofina actúa solo durante los procesos de aprendizaje y memoria ${ }^{(17)}$. Por otro lado, al determinarse el nivel de satisfacción media según atributos de cada una de las dimensiones de la calidad percibida, se volvió a evidenciar la importancia del entorno para un buen aprendizaje.

En cuanto a la dimensión, información recibida e integrada, el atributo con más alto nivel de satisfacción media le correspondió al profesor genera diálogos abiertos para la búsqueda de conocimiento a través de las intervenciones dándole al aprendizaje el rol protagóni- co, dado que el docente y el estudiante se ven influidos por la información y el entorno en el cual se aprende y por el órgano del aprendizaje, el cerebro ${ }^{(11)}$. Además, la memoria es más intensa, cuanto más frecuente, más intensa y más profunda es la manipulación de la información y los conocimientos ${ }^{(11)}$. Así mismo, el menor nivel de satisfacción media para los atributos el profesor presenta propuestas desafiantes que obliga esfuerzo intelectual y el profesor privilegia en la clase la formulación de preguntas se explicaría por el hecho que todo esfuerzo intelectual demanda más energía disponible del cual carecieron la mayoría de los estudiantes por solo haber desayunado en el día de la clase, lo que les condujo al agotamiento. Sumándose el uso de estrategias didácticas desfasadas de lo cotidiano que no hacen sino cansarlos y agotarlos ${ }^{(12)}$.

En la dimensión materiales didácticos, el hecho que el mayor nivel de satisfacción media le correspondiera al atributo el profesor incentiva el desarrollo del razonamiento intuitivo y el carácter lúdico de las actividades en el aula respondería a la práctica de ejercicios al inicio y en el intermedio de cada clase, lo que llevó a optimizar su disposición psíquica al esfuerzo cognitivo ${ }^{(19)}$, más aún cuanto a más acción más capacidad para recordar. Finalmente, el hecho que en la dimensión percepción del docente del atributo el profesor estimula a que el alumno construya imágenes mentales con el tema de la clase para lograr mantener la atención del alumno, incentivando a descubrir alguna característica del objeto de la clase, motivó que el nivel de satisfacción media fuera mayor, hecho que estaría sustentado en que la atención, imaginación, memorización y la comprensión son gestos mentales que facilitan al estudiante apropiarse del concepto principal de la clase, explorarlo y comprenderlo ${ }^{(12,14,24)}$. Además, respondió al movimiento, a la inflexión, tono y volumen cambiante de la voz del docente y a la gestualidad de las manos, y a la música utilizada durante la clase ${ }^{(12)}$, al activar el tálamo y el gyrus cínguli anterior.
Mientras el nivel de satisfacción media logrado en los atributos el profesor demanda de los estudiantes realizar simulaciones mentales de la característica más significativa del tema de la clase, y el profesor aprecia la importancia de las emociones de los educandos dado que es importante para el aprendizaje, se sustentaría en el hecho que la imitación y lo novedoso capturan la atención del hipocampo, así como la emoción positiva facilita almacenar lo aprendido en la memoria de largo plazo al incrementar la liberación de dopamina ${ }^{(4)}$. Además, la representación mental es necesaria e indispensable para iniciar las funciones del ejecutivo central ${ }^{(14)}$. Es la representación mental de las informaciones percibidas por el estudiante que la hace accesible a la conciencia al ser llevada de la forma audible a la visible, al transformar la información en objeto mental ${ }^{(14)}$. Conociendo que las emociones ${ }^{(12)}$ favorecen los procesos de atención, de recuerdo y toma de decisiones, se insistió en generar la emoción en los estudiantes para activar la zona del hipocampo y lograr que estudien en un estado de buen humor ${ }^{(11,18,25)}$.

Por otro lado, el sistema atencional supervisor actúa como el sistema ejecutivo central frente a tareas novedosas, activando los procesos de anticipación, selección de objetivos, planificación y control ${ }^{(19,25)}$. Precisamente, durante todo el proceso educativo se conversó con los estudiantes, se estimuló la memorización con imágenes visuales por el efecto estimulador del hipocampo y de la corteza parieto-témporo-occipital ${ }^{(2,18)}$, así como se incentivó el uso de la tormenta de ideas como estrategia para eliminar el miedo que limita los procesos creativos ${ }^{(11)}$.

Finalmente, los estudiantes le asignaron mayor prioridad a los atributos el profesor mantiene la atención del alumno y el profesor se esmera en el objeto de la clase, priorizando entre todos el conocimiento tiene relación con la realidad. Priorización importante que no solo permite la adecuada asignación de recursos destinados a mejorar la calidad 
percibida, sino también se debe a que el sistema atencional operativo es apropiado para la memoria de trabajo ${ }^{(21)}$, cuyo contenido es necesario para el accionar del ejecutivo central que realiza tareas cognitivas con la intervención de la memoria operativa para realizar operaciones de selección de estrategias y control independiente de la dinámica de interacción neuronal entre neuronas pre frontales y otras áreas corticales y subcorticales ${ }^{(2,25)}$.

Es más, para que se dé una actividad mental coherente, es indispensable el actuar del marcador somático (25), sugiriendo que los marcadores somáticos expresan a su vez las preferencias acumulativas que se han adquirido y recibido ${ }^{(19-21)}$. Así mismo, recuperar la información tiene que ver con seleccionar la adecuada información almacenada en la memoria de largo plazo, definir los objetivos a alcanzar, señalar lo que se debe guardar y precisar lo que se tiene que recuperar ${ }^{(22)}$. Por otro lado, se fijó el eje central de la clase en la novedad y en el significado del tema central de la clase para que el hipocampo al liberar dopamina lo almacene, creando una representación neuronal de ella ${ }^{(1)}$, siendo lo importante para el aprendizaje el valor anticipado de la gratificación de un estímulo ${ }^{(11)}$. En conclusión, la utilización de los conocimientos neurocientíficos en las clases mejoró la percepción de la calidad del servicio educativo reflejada en los niveles de satisfacción media y global.

\section{REFERENCIAS BIBLIOGRÁFICAS}

1. Benarós S, Lipina SJ, Segretin S, Hermida J, Colombo JA. Neurociencia y educación: hacia la construcción de puentes interactivos. Rev Neurol. 2010;50(3):179-86.

2. Campos L. Neuroeducación: uniendo las neurociencias y la educación en la búsqueda del desarrollo humano. Organización de Estados Americanos. La Educación. No 143. Junio 2010. Disponible en http://dragodsm.com.ar/pdf/ dragodsm-neurociencias-educacion-y-desarrollo06-2012.pdf.

3. Serra-Grabulosa JM, Adan A, Pérez-Pàmies M, Lachica J, Membrives S. Bases neurales del procesamiento numérico y del cálculo. Rev Neurol. 2010; 50 (1): 39-46.

4. Gudiño V. Desafío del SXXI: Neurociencia aplicada al Aprendizaje y la Educación. Boletín REDEM 2010. Disponible en http://www.redem.org/boletin/ boletin150910.html.

5. Aparicio X. Neurociencias y la transdisciplinariedad en la educación. Rev Univ de Inv y Diálogo Académico. 2009;5(2).

6. Pizarro B. Neurociencia y Educación. Aula Abierta. Madrid: Ed La Muralla S.A. 2003.

7. Cano F. Diferencias de género en estrategias y estilos de aprendizaje. Psicothema. 2000;12(3):360-7.

8. Medina J. Los 12 principios del cerebro. Una explicación sencilla de cómo funciona para obtener el máximo desempeño. Bogotá Colombia. Ed Norma. 2010.

9. Small G. El cerebro digital. Cómo las nuevas tecnologias están cambiando nuestra mente. Barcelona España. Ediciones Urano. 2009.

10. Spitzer M. Aprendizaje. Neurociencia y la escuela de la vida. Barcelona. Ediciones Omega S.A. 2005.

11. Ortiz T. Neurociencia y Educación. Madrid: Alianza Editorial, S.A. 2009:263 pp.

12. Blakemore S, Frith U. Cómo aprende el cerebro. Las claves para la Educación. Barcelona. Editorial Ariel S.A. 2008

13. Évano Ch. La gestión mental. Otra forma de ver y escuchar en pedagogia. Barcelona. Editorial GRAÓ. 2006

14. Ortiz T, Poch-Nroto J, Requena C, Santos JM, Martínez A, Barcia-Albacar JA. Neuroplasticidad cerebral en áreas occipitales en adolescentes ciegos. Rev Neurol. 2010;50(3):S19-S23.

15. Hernández-Muela S. Plasticidad neuronal funcional. Rev Neurol. 2004;38(1):S58-S68.
16. Gómez-Palacios A, Escobar-Rodríguez ML. Codificación y retención de la memoria: el factor neurotrópico derivado del cerebro (BDNF) en la plasticidad sináptica. Rev Neurol. 2007;45(7):40917.

17. Molero A. Sustratos neurales de la modulación contextual en el aprendizaje asociativo. Rev Neurol. 2008;47(2):83-8.

18. Tirapu J, Muñoz J, Pelegrin C. Funciones ejecutivas: necesidad de una integración conceptual. Rev Neurol. 2002;34(7):673-85.

19. Tirapu J, García A, Ríos M, Ardila A. Editores. Neuropsicología de la corteza frontal y las funciones ejecutivas. Barcelona. España: Ed Viguera Editores. 2012.

20. Tirapu-Ustárroz J, Muñoz-Céspedes J. Memoria y funciones ejecutivas. Rev Neurol. 2005;41(8):47584.

21. Machado S, Portella CE, Silva JG, Velasquez B, Bastos $\mathrm{NH}$ y col. Aprendizaje y memoria implícita: mecanismos y neuroplasticidad. RevNeurol. 2008;46(9):543-9.

22. Smith E, Kosslyn S. Procesos cognitivos: modelos y bases neuronales. Madrid: Pearson Educación, S.A. 2008

23. Cronin J, Taylor S. Measuring service quality: a reexamination and extension. J Marketing. 1992;56:5568. doi: http://dx.doi.org/10.2307/1252296

24. Quian R. Las neuronas de la conciencia. Rev Electrónica de Divulgación. 2008;2(2):47-9. Disponible en: www.le.ac.uk/neuroengineering y de http:// medina-psicologia.ugr.es.

25. Damasio AR. The somatic marker hypothesis and the possible functions of the prefrontal cortex. En: Roberts AC, Robbins TW, Weiskrantz L, Eds. The frontal cortex: executive and cognitive functions. New York: OxfordUniversity Press. 1998.

Artículo recibido el 25 de setiembre de 2013 y aceptado para publicación el 29 de enero de 2014.

Fuentes de financiamiento: Propias.

Conflicto de intereses: Los autores expresan no tener algún conflicto de interés.

Correspondencia:

Dr. Manuel H. Izaguirre Sotomayor

Facultad de Medicina Humana

Correo electrónico: manuel.izaguirre@gmail.com 